\title{
Artificial Intelligence to Improve the Food and Agriculture Sector
}

\author{
Rayda Ben Ayed $\mathbb{D D}^{1}$ and Mohsen Hanana $\mathbb{D}^{2}$ \\ ${ }^{1}$ Laboratory of Molecular and Cellular Screening Processes, Centre of Biotechnology of Sfax, P. B. 1177, Sfax 3018, Tunisia \\ ${ }^{2}$ Laboratory of Extremophile Plants, Biotechnology Center of Borj-Cédria, B. P. 901, Hammam Lif 2050, Tunisia
}

Correspondence should be addressed to Rayda Ben Ayed; raydabenayed@yahoo.fr

Received 19 February 2021; Revised 6 April 2021; Accepted 12 April 2021; Published 22 April 2021

Academic Editor: Rijwan Khan

Copyright (C) 2021 Rayda Ben Ayed and Mohsen Hanana. This is an open access article distributed under the Creative Commons Attribution License, which permits unrestricted use, distribution, and reproduction in any medium, provided the original work is properly cited.

\begin{abstract}
The world population is expected to reach over 9 billion by 2050, which will require an increase in agricultural and food production by $70 \%$ to fit the need, a serious challenge for the agri-food industry. Such requirement, in a context of resources scarcity, climate change, COVID-19 pandemic, and very harsh socioeconomic conjecture, is difficult to fulfill without the intervention of computational tools and forecasting strategy. Hereby, we report the importance of artificial intelligence and machine learning as a predictive multidisciplinary approach integration to improve the food and agriculture sector, yet with some limitations that should be considered by stakeholders.
\end{abstract}

\section{Introduction}

The estimation of the global food production must be increased by $60-110 \%$ to feed $9-10$ billion of the population by $2050[1,2]$. Thus, the sustainability of agriculture field is the key to guarantee food security and hunger eradication for the ever-growing population. In addition, due to the appearance of several food safety scandals and incidents in the food sector such as bovine spongiform encephalopathy and dioxin in poultry [3], a well-documented traceability system has become a requirement for quality control in the food chain. Moreover, weather and climate change conditions, together with the sustainable water management due to water scarcity, are crucial challenges in the next years. For these reasons, urgently, the establishment of a strategic shift from the current paradigm of enhanced agricultural productivity to agricultural sustainability is needed. To anticipate efficient solutions, helping farmers and stakeholders to enhance their decision by adopting sustainable agriculture practices is a crucial choice, especially the use of digital technologies including Internet of things (Iot), Artificial Intelligence (AI), and cloud computing. Additionally, the subsets of AI (machine and deep learning algorithms) combined with location intelligence technologies are extensively used. The goal of our review is to present the main applications of artificial intelligence and machine learning techniques in the agri-food sector.

\section{Artificial Intelligence and Machine Learning Approach}

The artificial intelligence (AI) is a creative tool that simulates the human intelligence and ability processes by machines, principally computer systems, robotics, and digital equipment [4]. Several applications of the AI include natural language processing (NLP) to comprehend human verbal communication as it is spoken, computer vision to see an analog-to-digital conversion such as video, and speech recognition and expert systems to simulate the judgment. The AI encoding is based on learning (acquire data and then create algorithms to turn them into actionable information), reasoning (choose the right algorithm to reach a preferred result), and self-correction (continually adjust designed algorithms and ensure that they provide the most accurate results) as three cognitive skills [5]. The AI technique is being used in several sectors which are seeing the fastest growth in the recent years such as finance, healthcare, retail, pharmaceutical research, intelligent process automation, and marketing. Machine learning (ML) is one of the central themes of AI and helps people to work more creatively and 
efficiently. In ML, statistical and mathematical methods are used to learn from datasets to make data-driven predictions/ decisions. Several different methods exist for this. General distinction can be made by two systems; the first one is the symbolic approaches (the induced rules and the examples are explicitly represented) and the second one is the subsymbolic approaches (artificial neuronal networks: ANN). The ML approach is classified into three major tasks: supervised, unsupervised, and reinforcement learning. According to the supervised learning, the aim of this approach is to map the variables to the preferred output variable [6]. The predictive model is created using the labeled data with the prior knowledge of the input and the desired output variables. Algorithms used under supervised learning techniques are numerous, particularly, decision trees, Bayesian networks, and regression analysis. Concerning the unsupervised learning, it includes algorithms such as Artificial Neural Networks (ANNs), clustering, genetic algorithm, and deep learning and uses unlabeled datasets without prior knowledge of the input and output variables. In fact and as mentioned by Jordan and Mitchell [7], this case of unsupervised machine learning method establishes the hidden patterns by using the unlabeled dataset and is primarily used for dimensionality reduction and exploratory data analysis. According to the third category of ML task named the reinforcement learning, numerous algorithms are used for machine skill acquisition, robot navigation, and real-time decision making such as Q-learning and deep Q-learning. In this case of ML task, the learner interacts with the environment to collect information and the two steps of training and testing datasets are combined. The learner gets awarded for his actions with the environment leading to an exploration versus exploitation dilemma. The learner must explore new unknown actions to gain more information as compared to exploiting the information already collected [8]. Recently, AI technology has opened the doors of its implementation in the agri-food sector. In fact, AI approaches offer significant contributions and assistances to understanding a model's identification, service creation, and the decision-making processes as support to the different agri-food's applications and supply chain stages. The principal goal of AI in agriculture is to provide precision and forecasting decision in order to improve the productivity with resource preservation [4]; through this, AI tools propose algorithms to evaluate performance, classify patterns, and to predict unexpected problems or phenomena in order to solve comprehension problems in the agricultural field and for the identification of pests and its suitable method of treatment, as well as the management of the irrigation process and water consumption by setting up smart irrigation systems. Abiotic and biotic factors are being assessed through remote sensing and sensors in order to optimize crop and livestock management $[4,9]$. In addition, the AI implementation and applications have enormous advantages which could revolutionize the agri-food sector and its related business. Firstly, AI provides more efficient ways to produce, harvest, and sell crops products as well as emphasis on checking defective crops and improving the potential for healthy crop production and also AI is being used in applications such as automated machine adjustments for weather forecasting and disease or pest identification with 98\% accuracy. In fact, recently, Sujatha et al. [10] compared the performance of machine learning (ML) and deep learning (DL) methods to detect and identify the citrus plant leaf disease. They showed that the VGG-16 deep learning method gave the best result in terms of disease classification accuracy. Secondly, the progression in the AI technique has reinforced agro-based businesses to run more proficiently by improving crop management practices, thus helping many tech businesses invest in algorithms that are becoming useful in agriculture as well as by solving the contrasts farmers face such as climate variation and an infestation of pests and weeds that decreases yields. Indeed, Crane-Droesch [11] developed a novel modeling approach for augmenting parametric statistical models with deep neural networks, which we term semiparametric neural networks (SNNs), and by using data on corn yield from the US Midwest, they showed the outperformance of this approach in predicting yields of years withheld during model training compared to classical statistical methods and fully nonparametric neural networks. Thirdly, by using AI tools, farmers could be able to remain updated with the data related to weather forecasting and, therefore, predicted weather data help farmers to increase yields and profits without risking the crop, and as a result, after analyzing the generated data, AI allows the farmers to better understand and learn and then to take the precaution by implementing practices in order to make a punctual smart decision. In fact, Fente and Singh [12] collected different weather parameters (temperature, precipitation, wind speed, pressure, dew point visibility, and humidity) from the Indian climate data center and implemented a weather forecasting model by using a recurrent neural network (RNN) with the long-short-term memory (LSTM) technique. They concluded that the used technique gave high-accuracy results compared to other weather forecasting approaches. Fourthly, AI approaches are capable of monitoring soil health and management by conducting and identifying the possible defects and nutrient deficiencies in the soil either by image captured with the camera recognition tool or by deep learning based tool to analyse flora patterns in farms and to simultaneously understand soil defects, plant pests, and diseases. In fact, Suchithra and Pai [13] classified and predicted the soil fertility indices and $\mathrm{pH}$ levels of Kerala north central laterite Indian region soil by using the Extreme Learning Machine (ELM) technique with different activation functions such as hard limit, sinesquared, triangular basis, hyperbolic tangent, and gaussian radial basis. They revealed that the maximum performance ( $80 \%$ of the accuracy rate calculations in every problem) for four out of five problems was obtained with the Gaussian radical basis function followed by hyperbolic tangent. However, the best performance $(90 \%)$ of the $\mathrm{pH}$ classification problem was given by the hyperbolic tangent, whereas the moderate values were given by the gaussian radial basis. Fifthly, an important functional benefit of the AI technology employment is the environmental protection by decreasing pesticide usage. For example and in order to manage weeds faster and with greater accuracy, AI techniques by 
implementing robotics, computer vision, and machine learning could help farmers to spray chemicals only where the weeds are; thus, this directly reduced the use of the chemical substance spraying on the whole field. Consequently, AI tools are helping farmers find more efficient actions to protect their crops from weeds. Finally, the practice of the advanced AI-based technologies has other advantages on the agri-food supply chain such as reducing employee training costs, reducing the time needed to solve problems, reducing the amount of human errors, reducing human intervention, and offering an automated good, accurate, and robust decision-making on the right time with low cost [14].

\section{Artificial Intelligence Technology and Application to Improve Agriculture and Food Industries}

Currently, the use of ML algorithms in the main four clusters (preproduction, production, processing, and distribution) of the agriculture supply chain is becoming more and more important [15]. In fact, in the preproduction step, the ML technologies are used, especially for the prediction of crop yield, soil properties, and irrigation requirements. In the next stage of the production phase, the ML could be used for disease detection and weather prediction. Concerning the third cluster of the processing phase, utilization of ML approaches is applied, especially to estimate of the production planning to reach a high and safe quality of the product. ML algorithms could be used also to the distribution cluster, especially in storage, transportation, and consumer analysis. The preproduction cluster is the initial step in the agriculture supply chain. It mainly concerns the prediction of crop yield, soil properties, and irrigation requirement. Many researchers report the importance of the crop yield production in order to better support plant management. In fact, by using as an input data (equipment requirements, nutrients, and fertilizers) in predicting efficient models based on ML algorithms, these precision agriculture tools aim to make stakeholders and farmers support ideal decision in crop yield forecasting and improve the smart farming practices. Recently, different ML algorithms are used for crop yield prediction such as the Bayesian network, regression, decision tree, clustering, deep learning, and ANN [16-18]. According to the prediction of soil management properties, several ML algorithms are used in learning soil properties. Among them, the LS-SVM (leastsquares support vector machine) method was used by Morellos et al. [19] to study 140 soil samples. Nahvi et al. [20] used the SaE (self-adpative evolutionary) ML algorithm to boost the performance of the extreme learning machine (ELM) architecture to estimate daily soil temperature. Additionally, Kumar et al. [21] proposed a novel method named the CSM (Crop Selection Method) to resolve crop selection problems and help improve net yield rate of crops over the season. In addition, Ben Ayed et al. [16] analyzed 18 worldwide table olive cultivars by using morphological, biological, and physicochemical parameters and the
Bayesian network to study the influence of these parameters in tolerance, productivity, and oil content. They revealed that oil content was highly influenced by the tolerance of the crop. Another important parameter in the preproduction cluster is the irrigation management that plays a crucial role in affecting the quality and quantity of the crops. In fact, to achieve an effective irrigation system (better decision in when, where, and how much to irrigate), researchers used soil moisture data, precipitation data, evaporation data, and weather forecasts as input data for simulation and optimization of predicted models based on ML adequate algorithms [22]. In fact, Arvind et al. [23] demonstrated that using ML algorithm associated with other technologies such as sensors, Zigbee, and Arduino microcontroller was efficient for prediction and tackles drought situations. In addition, Cruz et al. [24] exploited the ANN feed-forward and back-propagation technologies to optimize the water resources in a smart farm. More recently, Choudhary et al. [25] used PLSR and other regression algorithms as an artificial intelligence tool combined with sensors for data collection and Internet of things hardware implementation to increase efficiency and economic feasibility.

The production cluster is the second phase in the agriculture supply chain. There are numerous parameters that affect and play a key role in the crop production step. Among them are the weather forecasts (sunlight, rainfall, humidity, etc.), crop protection against biotic stress factors (weeds and pathogens) and abiotic stress factors (nutrient and water deficiency), crop quality management, and harvesting. Many different ML algorithms are used to simulate effective models for weather prediction (ANN, deep learning, decision tree, ensemble learning, and instance-based learning) [26], for crop protection (clustering and regression) [27], ANN, deep learning [14], weed detection (ANN, decision tree, deep learning, and instance-based learning) [28], crop quality management (clustering and regression) [29], and harvesting (deep neural networks, data mining techniques such as $k$ mean clustering, $k$ nearest neighbor, ANN, and SVM) [30]. During the harvest stage which is the final horticultural stage after the ripening of the crops, ML algorithms are also used to predict the transformation of the fruit or crop color. In fact, many research teams used ML algorithms to predict the fruit ripening stages and fruit maturity such as Gao et al. [31] who achieved $98.6 \%$ classification accuracy when they used hyperspectral datasets and the AlexNet CNN deep learning model to classify the strawberry fruits into early-ripe and ripe stages. The processing cluster is the third stage in the agriculture supply chain. There are many types of processing techniques of agriculture products such as heating, cooling, milling, smoking, cooking, and drying. The choice of effective combined parameters in the processing stage results in a high quality and quantity of food product and, at the same time, avoiding overutilization of resources. To achieve this goal, several food industries use modern food processing technologies by installing software algorithms based on ML. Among the used ML algorithms, there are genetic algorithm, ANN, clustering, and Bayesian network [32]. In fact, Arora and Mangipudi [33] proposed support vector machine 
(SVM) classifier and artificial neural network (ANN) models to detect the presence of nitrosamine in the red meat food samples, and the obtained predictive modeling results revealed that the highest testing accuracy was obtained using the deep learning model. Additionally, Farah et al. [34] used differential scanning calorimetry combined with ML tools (such as gradient boosting machine, random forest, RF, multilayer perceptron, MLP, and GBM) to determine the milk characteristics and authenticity and to detect fraud. The most efficient results were obtained with GBM and MLP machine learning tools which were able to classify $100 \%$ of adulterated samples. The distribution cluster is the final step in the agriculture supply chain. This stage is the connection between food production and processing and the final use or final consumer. ML algorithms could be used in storage, transportation, consumer analytics, and inventory management. In transportation and storage steps, the mainly used algorithms are genetic algorithm, clustering, and regression. These predictive techniques aim to better preserve the food product quality, to ensure safe food products and to minimize the product damage by tracing the product [16]. For the consumer analytics, ML techniques such as deep learning and ANN are used in the food retailing phase for predicting consumer demand, perception, and buying behavior. For the inventory management, the use of ML genetic algorithms helps in predicting daily demand and to ensure that there are no inventory-related problems [35]. Examples of AI-applied technology are numerous in the agri-food sector, i.e., robotics and mechatronics [2], drones $[2,36]$, geographic information systems (GISs) [37], blockchain (BC) [38], and satellite guidance [2]. Miranda et al. [39] have reported and described these items as sensing, smart, and sustainable technologies, providing systematic process where connectivity, automation, precision, monitoring, and digitization are prevalent [40-43]. Smart mechanization, robotics, and mechatronics in agriculture aim to reduce drudgery and minimize inputs using highly autonomous and intelligent machines [2]. From horse to tractor, robots, and intelligent vehicles, a revolutionary era has come for agriculture and food industry, from rudimentary to high efficiency of agriculture with the introduction of mechanization, innovative technologies, computerized analysis, and decision, improving farming activities and crop productivity [2]. Revolutionizing machines, often called "agribots," are now used in agriculture for all kinds of activities, namely, soil preparation, seed sowing, weed and pest treatment, irrigation, fertilization, and ultimately, grain and fruit harvesting, minimizing effort and energy cost [2,44-47]. As a whole crop management, agricultural drones can be used starting from soil treatment (herbicide), going to sowing step, plant treatment (pesticide), and physiological control and observation, and ending with harvest time determination [2, 36, 48-51]. Agricultural drones are now able to supply water, fertilizers, herbicides, and pesticides and even film, capture images, and generate maps in real time of plants and field in order to help farmers take management decision $[2,48,52-56]$. Today, farmers use drones for livestock surveillance for monitoring illnesses, injuries, and even pregnancies [57]. In 2019, the worldwide agriculture drone market value was about USD one million and is expected to reach USD 3.7 million by 2027 [58], whereas the robot and agriculture drone market is projected to reach USD 23 billion by $2028[59,60]$. Based on geospatial technology that relies on satellite, GIS is applied on several fields of agriculture: crop management, irrigation control, yield estimation, disease and weed control, farming automation, livestock monitoring, vegetation mapping, erosion, and land degradation forecast [37, 61-68]. Application of GIS is, therefore, suitable in precision agriculture, real-time control, and raising awareness and significantly contributes to meet the needs of continuous rise in food demand. Blockchain is another technology that answers to consumer's awareness about food origin, quality, and mainly, safety. BC affords transparency, trust, certification, and traceability of food product supply chain from farm to fork, where every single operation and data are timely registered, saved, encrypted, and secured, not in a single central server nor under a single control, but in a common platform database where every user could access and take part in transactions [38, 69-79]. Such digital and computerized traceability of the whole food supply chain would allow detection of deficiency, contamination, and adulteration of the product, thus optimizing its quality and safety; therefore, a multitude of agencies, consortia, and platforms were born in this context [79]. In 2020, the worldwide market of BC in agri-food market size was about USD 133 million; it is estimated to grow and reach around USD 950 million by 2025 [69]. Satellite-guided technology applied to agriculture improved farm monitoring and aided mapping agricultural zones, soil management, crop husbandry, irrigation disease and weed control, yield estimation, and harvesting $[2,80-88]$. Hence, in the late 1900 s, one single farmer was able to produce food grains for 128 , actually, and in the future, through smart agriculture, this ratio will greatly increase [2].

\section{Limits and Drawbacks of AI and ML}

However, despite all these advantages, the AI technology has also some drawbacks representing challenges. Firstly, the most important social challenge is the unemployment that could be a threat; in fact, smart machines and robots could replace the majority of the repetitive works and tasks; thus, human interference is becoming less, which will cause a major problem in the employment standards. Other technological challenges, for instance, machines can do only those tasks which they are programmed or developed to do, and anything out of that they tend to crash or give irrelevant outputs could be a major backdrop. In addition, the high costs of creation and maintenance of the smart machines as well as the cleaver computers could be considered as technological limits of the AI technologies, especially that AI is updating every day which is why the hardware and software need to get updated with time to meet the latest requirements. Machines need repairing and maintenance which is expensive. The creation requires huge costs as they are very complex machines. Other issues related to these applications are their high cost that could increase the price 
of the products. Moreover, beyond the opportunities afforded by smart and computerized technologies, some risks and apprehensions could be posed for sustainability, particularly the massive energy consumption, e-waste problem, market concentration, job displacement, and even the ethical framework $[79,89]$.

\section{Conclusions}

The agriculture and food industries are one of the most vital fields for humanity. The first products of agriculture are used as inputs in several multiactor distributed supply chains, including four clusters or stages of the agriculture supply chain (preproduction, production, processing, and distribution) in order to reach the end user or consumer. Due to several challenges in the future for the agriculture and food sector and various factors such as climate change, population growth, technological progress, and the state of natural resources (water, etc.), it is urgent to use the digital technologies at different stages of agriculture supply chain such as automation of farm machinery, use of sensors and remote satellite data, artificial intelligence, machine learning for improved monitoring of crops, and water, for agriculture food product traceability. In the present study, we demonstrate the main applications of the $\mathrm{AI}$ and $\mathrm{ML}$ algorithms in different clusters of the agriculture supply chain and the unquestionable growing tendency in the adoption of these algorithms to improve food industries.

\section{Data Availability}

The data used to support the findings of this study are available from the corresponding author upon request.

\section{Conflicts of Interest}

The authors declare no conflicts of interest.

\section{References}

[1] J. Rockström, J. Williams, G. Daily et al., "Sustainable intensification of agriculture for human prosperity and global sustainability," Ambio, vol. 46, no. 1, pp. 4-17, 2017.

[2] K. R. Krishna, Push Button Agriculture: Robotics, Drones, Satellite-Guided Soil and Crop Management, Apple Academic Press, Waretown, NJ, USA, 2016.

[3] R. Ben-Ayed, N. Kamoun-Grati, and A. Rebai, "An overview of the authentication of olive tree and oil," Comprehensive Reviews in Food Science and Food Safety, vol. 12, pp. 218-227, 2013.

[4] G. S. Patel, A. Rai, N. N. Das, and R. P. Singh, Eds., Smart Agriculture: Emerging Pedagogies of Deep Learning, Machine Learning and Internet of Things, CRC Press, Boca Raton, FL, USA, 1st edition, 2021.

[5] A. Gharaei, M. Karimi, and S. A. Hoseini Shekarabi, "An integrated multi-product, multi-buyer supply chain under penalty, green, and quality control polices and a vendor managed inventory with consignment stock agreement: the outer approximation with equality relaxation and augmented penalty algorithm," Applied Mathematical Modelling, vol. 69, pp. 223-254, 2019.
[6] B. B. Traore, B. Kamsu-Foguem, and F. Tangara, "Data mining techniques on satellite images for discovery of risk areas," Expert Systems with Applications, vol. 72, pp. 443-456, 2017.

[7] M. I. Jordan and T. M. Mitchell, "Machine learning: trends, perspectives, and prospects," Science, vol. 349, 2015.

[8] M. Mohri, A. Rostamizadeh, and A. Talwalkar, Foundations of Machine Learning, MIT Press, Cambridge, MA, USA, 2018, https://cs.nyu.edu/ mohri/mlbook/.

[9] A. Suprem, N. Mahalik, and K. Kim, "A review on application of technology systems, standards and interfaces for agriculture and food sector," Computer Standards \& Interfaces, vol. 35, no. 4, pp. 355-364, 2013.

[10] R. Sujatha, J. M. Chatterjee, N. Jhanjhi, and S. N. Brohi, "Performance of deep learning vs machine learning in plant leaf disease detection," Microprocessors and Microsystems, vol. 80, p. 103615, 2021.

[11] A. Crane-Droesch, "Machine learning methods for crop yield prediction and climate change impact assessment in agriculture," Environmental Research Letters, vol. 13, no. 11, p. 114003, 2018.

[12] D. N. Fente and D. K. Singh, "Weather forecasting using artificial neural network," in Proceedingsof the 2018 Second International Conference on Inventive Communication and Computational Technologies (ICICCT), Coimbatore, India, April 2018.

[13] M. S. Suchithra and M. L. Pai, "Improving the prediction accuracy of soil nutrient classification by optimizing extreme learning machine parameters," Information Processing in Agriculture, vol. 7, no. 1, pp. 72-82, 2020.

[14] A. Kamilaris and F. X. Prenafeta-Boldú, "Deep learning in agriculture: a survey," Computers and Electronics in Agriculture, vol. 147, pp. 70-90, 2018.

[15] O. Ahumada and J. R. Villalobos, "Application of planning models in the agri-food supply chain: a review," European Journal of Operational Research, vol. 196, no. 1, pp. 1-20, 2009.

[16] R. Ben Ayed, K. Ennouri, F. Ben Amar, F. Moreau, M. A. Triki, and A. Rebai, "Bayesian and phylogenic approaches for studying relationships among table olive cultivars," Biochemical Genetics, vol. 55, no. 4, pp. 300-313, 2017.

[17] D. Elavarasan, D. R. Vincent, V. Sharma, A. Y. Zomaya, and K. Srinivasan, "Forecasting yield by integrating agrarian factors and machine learning models: a survey," Computers and Electronics in Agriculture, vol. 155, pp. 257-282, 2018.

[18] C. Zhang, J. Liu, J. Shang, and H. Cai, "Capability of crop water content for revealing variability of winter wheat grain yield and soil moisture under limited irrigation," Science of The Total Environment, vol. 631-632, pp. 677-687, 2018.

[19] A. Morellos, X.-E. Pantazi, D. Moshou et al., "Machine learning based prediction of soil total nitrogen, organic carbon and moisture content by using VIS-NIR spectroscopy," Biosystems Engineering, vol. 152, pp. 104-116, 2016.

[20] B. Nahvi, J. Habibi, K. Mohammadi, S. Shamshirband, and O. S. Al Razgan, "Using self-adaptive evolutionary algorithm to improve the performance of an extreme learning machine for estimating soil temperature," Computers and Electronics in Agriculture, vol. 124, pp. 150-160, 2016.

[21] R. Kumar, M. P. Singh, P. Kumar, and J. P. Singh, "Crop Selection Method to maximize crop yield rate using machine learning technique," in Proceedings of the 2015 International Conference on Smart Technologies and Management for Computing, Communication, Controls, Energy and Materials (ICSTM), pp. 138-145, Avadi, India, May 2015.

[22] A. Goap, D. Sharma, A. K. Shukla, and C. Rama Krishna, "An IoT based smart irrigation management system using 
Machine learning and open source technologies," Computers and Electronics in Agriculture, vol. 155, pp. 41-49, 2018.

[23] G. Arvind, V. G. Athira, H. Haripriya, R. A. Rani, and S. Aravind, "Automated irrigation with advanced seed germination and pest control," in Proceedings of the 2017 IEEE Technological Innovations in ICT for Agriculture and Rural Development (TIAR), pp. 64-67, Chennai, India, April 2017.

[24] J. R. Dela Cruz, R. G. Baldovino, A. A. Bandala, and E. P. Dadios, "Water usage optimization of smart farm automated irrigation system using artificial neural network," in Proceedings of the 2017 5th International Conference on Information and Communication Technology (ICoIC7), pp. 1-5, Melaka, Malaysia, May 2017.

[25] V. Gaurav, S. Choudhary, A. Singh, and S. Agarwal, "Autonomous crop irrigation system using artificial intelligence," International Journal of Engineering and Advanced Technology (IJEAT), vol. 8, 2019.

[26] M. K. Saggi and S. Jain, "Reference evapotranspiration estimation and modeling of the Punjab Northern India using deep learning," Computers and Electronics in Agriculture, vol. 156, pp. 387-398, 2019.

[27] A. Singh, N. Shukla, and N. Mishra, "Social media data analytics to improve supply chain management in food industries," Transportation Research Part E: Logistics and Transportation Review, vol. 114, pp. 398-415, 2018.

[28] K. Liakos, P. Busato, D. Moshou, S. Pearson, and D. Bochtis, "Machine learning in agriculture: a review," Sensors, vol. 18, no. 8, p. 2674, 2018.

[29] A. Chlingaryan, S. Sukkarieh, and B. Whelan, "Machine learning approaches for crop yield prediction and nitrogen status estimation in precision agriculture: a review," Computers and Electronics in Agriculture, vol. 151, pp. 61-69, 2018.

[30] E. J. Sadgrove, G. Falzon, D. Miron, and D. W. Lamb, "Realtime object detection in agricultural/remote environments using the multiple-expert colour feature extreme learning machine (MEC-ELM)," Computers in Industry, vol. 98, pp. 183-191, 2018.

[31] Z. Gao, Y. Shao, G. Xuan, Y. Wang, Y. Liu, and X. Han, "Realtime hyperspectral imaging for the in-field estimation of strawberry ripeness with deep learning," Artificial Intelligence in Agriculture, vol. 4, pp. 31-38, 2020.

[32] X. Ma, S. Wang, and Q. Bai, "Coordination of production scheduling and vehicle routing problems for perishable food products," International Journal of Internet Manufacturing and Services, vol. 6, no. 1, p. 79, 2019.

[33] M. Arora and P. Mangipudi, "A computer vision-based method for classification of red meat quality after nitrosamine appendage," International Journal of Computational Intelligence and Applications, vol. 20, no. 1, p. 2150005, 2021.

[34] J. S. Farah, R. N. Cavalcanti, J. T. Guimarães et al., "Differential scanning calorimetry coupled with machine learning technique: an effective approach to determine the milk authenticity," Food Control, vol. 121, p. 107585, 2021.

[35] A. Dolgui, M. K. Tiwari, Y. Sinjana, S. K. Kumar, and Y.-J. Son, "Optimising integrated inventory policy for perishable items in a multi-stage supply chain," International Journal of Production Research, vol. 56, no. 1-2, pp. 902-925, 2018.

[36] K. R. Krishna, Agricultural Drones: A Peaceful Pursuit/K, Apple Academic Press, Palm Bay, FL, USA, 2017.

[37] S. Ravensberg, "GIS in agriculture, integrate sustainability," 2018, https://www.integratesustainability.com.au/2018/11/23/ gis-in-agriculture/.
[38] G. Zhao, S. Liu, C. Lopez et al., "Blockchain technology in agri-food value chain management: a synthesis of applications, challenges and future research directions," Computers in Industry, vol. 109, pp. 83-99, 2019.

[39] J. Miranda, P. Ponce, A. Molina, and P. Wright, "Sensing, smart and sustainable technologies for agri-food 4.0," Computers in Industry, vol. 108, pp. 21-36, 2019.

[40] L. Trivelli, A. Apicella, F. Chiarello, R. Rana, G. Fantoni, and A. Tarabella, "From precision agriculture to Industry 4.0," British Food Journal, vol. 121, no. 8, pp. 1730-1743, 2019.

[41] M. Bacco, P. Barsocchi, E. Ferro, A. Gotta, and M. Ruggeri, "The digitisation of agriculture: a survey of research activities on smart farming," Array, vol. 3-4, p. 100009, 2019.

[42] M. J. O'Grady, D. Langton, and G. M. P. O'Hare, "Edge computing: a tractable model for smart agriculture?" Artificial Intelligence in Agriculture, vol. 3, pp. 42-51, 2019.

[43] K. Jha, A. Doshi, P. Patel, and M. Shah, "A comprehensive review on automation in agriculture using artificial intelligence," Artificial Intelligence in Agriculture, vol. 2, pp. 1-12, 2019.

[44] G. Kootstra, X. Wang, P. M. Blok, J. Hemming, and E. van Henten, "Selective harvesting robotics: current research, trends, and future directions," Current Robotics Reports, vol. 2, no. 1, pp. 95-104, 2021.

[45] K. Sennaar, "Agricultural robots-present and future applications,” 2021, https://emerj.com/ai-sector-overviews/ agricultural-robots-present-future-applications/.

[46] C. W. Bac, E. J. Van Henten, J. Hemming, and Y. Edan, "Harvesting robots for high-value crops: state-of-the-art review and challenges ahead," Journal of Field Robotics, vol. 31, 2014.

[47] A. Zujevs, V. Osadcuks, and P. Ahrendt, "Trends in robotic sensor technologies for fruit harvesting: 2010-2015," Procedia Computer Science, vol. 77, 2015.

[48] J. Huuskonen and T. Oksanen, "Soil sampling with drones and augmented reality in precision agriculture," Computers and Electronics in Agriculture, vol. 154, pp. 25-35, 2018.

[49] "Importance of drone technology in Indian agriculture, farming," 2021, https://www.equinoxsdrones.com/blog/importanceof-drone-technology-in-indian-agriculture-farming.

[50] "Drones and precision agriculture: the future of farming," 2021, https://www.microdrones.com/en/content/drones-andprecision-agriculture-the-future-of-farming/.

[51] How Drones Can Help Manage the World's Food Supply, World Economic Forum, Cologny, Switzerland, 2021, https://www. weforum.org/agenda/2018/09/how-drones-can-manage-the-fo od-supply-chain-and-tell-you-if-what-you-eat-is-sustainable/.

[52] Agricultural Drones: A Peaceful Pursuit, Routledge \& CRC Press, Boca Raton, FL, USA, 2021, https://www.routledge.com/ Agricultural-Drones-A-Peaceful-Pursuit/Krishna/p/book/9781 774636428.

[53] How Drones are Being Used in Australia to Make Farming More Efficient, ForestTECH, Rotorua, New Zealand, 2021, https:// foresttech.events/how-drones-are-being-used-in-australia-to-ma ke-farming-more-efficient/.

[54] Robots and Drones: Addressing Agriculture's Long-Term Challenges, IDTechEx, Cambridge, UK, 2018, https://www. idtechex.com/fr/research-article/robots-and-drones-addressi ng-agricultures-long-term-challenges/14126.

[55] Drones in Agriculture Industry Helps with Demand for Food Production, ITChronicles, Montreal, Canada, 2020, https:// itchronicles.com/artificial-intelligence/game-of-drones-in-bigdata-and-agriculture-industry/. 
[56] Robots Agricoles, Drones et IA : 2020-2040 : Technologies, Marchés et Joueurs, https://www.idtechex.com/fr/researchreport/agricultural-robots-drones-and-ai-2020-2040-techn ologies-markets-and-players/749, Cambridge, UK, IDTechEx.

[57] T. Jennings, Farming Drones: the Future of Agriculture?, CropLife, Brussels, Belgium, 2017, https://www.croplife.com/ iron/farming-drones-the-future-of-agriculture/.

[58] Agriculture Drone Market Size, Share, Industry Analysis, 2027, Fortune Business Insights, Pune, Maharashtrahttps://www. fortunebusinessinsights.com/agriculture-drones-market102589.

[59] BIS Research, The Rising Demand for Agriculture Drones and Robots, BIS Research, Fremont, CA, USA, 2021, https://blog. marketresearch.com/the-rising-demand-for-agriculturedrones-and-robots.

[60] Global Agriculture Drones and Robots Market: Focus on Drones, Robot Type (Milking Robot, Harvesting \& Picking Robot, Autonomous Robot Tractor), Farm Produce, Farming Environment, Business Model, Regulations, and Patents-Analysis \& Forecast, 2018-2028, BIS Research, Fremont, CA, USA, 2021, https://www.marketresearch.com/BISResearch-v4011/Global-Agriculture-Drones-Robots-Focus$12393359 /$ ?progid $=91587$.

[61] GIS Applications in Agriculture, Routledge \& CRC Press, Boca Raton, FL, USA, 2021, https://www.routledge.com/GISApplications-in-Agriculture/Pierce-Clay/p/book/ 9780849375262.

[62] GIS Applications in Agriculture, Routledge \& CRC Press, Boca Raton, FL, USA, 2021, https://www.routledge.com/GISApplications-in-Agriculture/book-series/GIS.

[63] GIS Applications in Agriculture, Volume 1, CRC Press, Boca Raton, FL, USA, 2021, https://www.nhbs.com/gisapplications-in-agriculture-volume-1-book.

[64] GIS Applications in Agriculture, Volume 2: in Nutrient Management for Energy Efficiency, Washington State University, Prosser, WA, USA, 2021, https://www.nhbs.com/gisapplications-in-agriculture-volume-2-book.

[65] GIS Applications in Agriculture, Volume 3: Invasive Species, CRC Press, Boca Raton, FL, USA, 2021, https://www.nhbs. com/gis-applications-in-agriculture-volume-3-book.

[66] GIS Applications in Agriculture, Volume 4: Conservation Planning, CRC Press, Boca Raton, FL, USA, 2021, https:// www.nhbs.com/gis-applications-in-agriculture-volume-4book.

[67] Use of GIS in Agriculture, Cornell Small Farms, Ithaca, NY, USA, 2017, https://smallfarms.cornell.edu/2017/04/use-ofgis/.

[68] GIS in Agriculture as the Key to Effective Decision-Making, Intellias, Lviv, Ukraine, 2020, https://www.intellias.com/gisin-agriculture/.

[69] Blockchain in Agriculture: Solving Agri-Food Supply Chain, Farrelly Mitchell, Dubai, UAE, 2020, https://farrellymitchell. com/blockchain-in-agriculture-solving-food-supply-chain/.

[70] F. Antonucci, S. Figorilli, C. Costa, F. Pallottino, L. Raso, and P. Menesatti, "A Review on blockchain applications in the agri-food sector," Journal of the Science of Food and Agriculture, vol. 99, 2019.

[71] M. P. Caro, M. S. Ali, M. Vecchio, and R. Giaffreda, "Blockchain-based traceability in agri-food supply chain management: a practical implementation," in Proceedings of the 2018 IoT Vertical and Topical Summit on AgricultureTuscany (IOT Tuscany), pp. 1-4, Tuscany, Italy, May 2018.
[72] A. M. G. Lamparte, Use of Blockchain Technologies in the AgriFood Sector, EIP-AGRI-European Commission, Brussels, Belgium, 2020, https:/ec.europa.eu/eip/agriculture/en/event/ use-blockchain-technologies-agri-food-sector (accessed April 4, 2021).

[73] G. A. Motta, B. Tekinerdogan, and I. N. Athanasiadis, "Blockchain applications in the agri-food domain: the first wave," Frontiers in Blockchain, vol. 3, 2020.

[74] M. Tripoli and J. Schmidhuber, Emerging Opportunities for the Application of Blockchain in the Agri-Food Industry, FAO and ICTSD, Rome, Italy, 2018.

[75] SmartAgriFood-Blockchain AgriOpenData, SmartAgriFood, Brussels, Belgiumhttps://www.smartagrifood.it/index-en. html.

[76] 2021 Blockchains in agrifood project, TNO. /projects/ agrifood.

[77] "Blockchain in agriculture and food supply chain market size, industry statistics, trends and analysis. COVID-19 impact analysis," 2021, https://www.marketsandmarkets.com/MarketReports/blockchain-agriculture-market-and-food-supply-chain55264825.html?gclid=CjwKCAjwx6WDBhBQEiwA_dP8rb1pY wZtfgPGY6Q7ITPmnCa5zDe7yJliCkiWjDEZtKbGWInzZh6p UBoCAdEQAvD_BwE.

[78] Blockchain in Agrifood: A Great Opportunity. . . Disguised as a Trend?, AgFunderNews, San Francisco, CA, USA, 2021.

[79] N. Patelli and M. Mandrioli, "Blockchain technology and traceability in the agrifood industry," Journal of Food Science, vol. 85, no. 11, pp. 3670-3678, 2020.

[80] Satellite Guidance, CropWatch, Beijing, China, 2015, https:// cropwatch.unl.edu/ssm/guidance.

[81] Digital Cameras, Remote Satellites Measure Crop Water Demand, EurekAlert Science News, Washington, DC, USA, 2021, https://www.eurekalert.org/pub_releases/2008-07/asfhdcr071708.php.

[82] A. I. De Castro, F. López-Granados, and M. Jurado-Expósito, "Broad-scale cruciferous weed patch classification in winter wheat using QuickBird imagery for in-season site-specific control," Precision Agriculture, vol. 14, no. 4, pp. 392-413, 2013.

[83] "Ground-based remote sensing system for irrigation scheduling-sciencedirect," 2021, https://www.sciencedirect.com/ science/article/abs/pii/S1537511012001523.

[84] "New satellite data will help farmers facing drought-climate change: vital signs of the planet," 2021, https://climate.nasa. gov/news/1145/new-satellite-data-will-help-farmers-facingdrought/.

[85] N. C. Coops and T. R. Tooke, "Introduction to remote sensing," in Learning Landscape Ecology: A Practical Guide to Concepts and Techniques, S. E. Gergel and M. G. Turner, Eds., Springer, New York, NY, USA, pp. 3-19, 2017.

[86] Precision Ag: Applying Agronomy Innovatively in Production Crop Consulting, Crop Quest, Dodge City, KS, USA, 2021, https://www.cropquest.com/category/precision-ag/.

[87] NASA, NASA Uses Satellite to Unearth Innovation in Crop Forecasting, NASA, Washington, DC, USA, 2021, https:// www.nasa.gov/topics/earth/features/crop_forecast.html.

[88] 2021 Home GDA. https://www.gdacorp.com.

[89] GAG, Digitising Agrifood, BCFN Foundation, Parma, Italy, 2021, https://www.barillacfn.com/en/publications/digitisingagrifood/. 